\title{
Chemoselective oxidation of 3-acetyl-2,3-dihydrobenzothiazoles by dimethyldioxirane
}

\author{
Albert Lévai ${ }^{a *}$ and József Jekő ${ }^{b}$ \\ ${ }^{a}$ Department of Organic Chemistry, University of Debrecen, Egyetem tér 1, H-4010 Debrecen, \\ Hungary, ${ }^{\mathrm{b}}$ ICN Hungary Co. Ltd., H-4440 Tiszavasvári, Hungary \\ E-mail: $\underline{\text { alevai@tigris.klte.hu }}$
}

Dedicated to Professor Gábor Bernáth on the occasion of his $70^{\text {th }}$ birthday

(received 09 Jan 03; accepted 13 Jan 03; published on the web 05 Feb 03)

\begin{abstract}
3-Acetyl-2,3-dihydrobenzothiazoles 11-20 have been prepared by the ring contraction of 2,4diaryl-2,3-dihydro-1,5-benzothiazepines 1-10 under acetylating conditions. Compounds 11,15-24 have been oxidized with isolated dimethyldioxirane (in acetone solution) at ambient temperature to afford 3-acetyl-2,3-dihydrobenzothiazole 1,1-dioxides $\mathbf{2 5 - 3 5}$ as sole isolable products.
\end{abstract}

Keywords: 2,4-Diaryl-2,3-dihydro-1,5-benzothiazepines, dimethyldioxirane, ring contraction

\section{Introduction}

In the course of our studies on the chemical transformations of the 2,4-diaryl-2,3-dihydro-1,5benzothiazepines, we have discovered their previously unprecedented ring contraction providing 2,2-disubstituted 3-acetyl-2,3-dihydrobenzothiazoles under acetylating conditions. ${ }^{1}$ Later, we have generalized this simple and convenient procedure for the preparation of related 2,2disubstituted 3-acyl-2,3-dihydrobenzothiazoles under acylating condtions by using various acid anhydrides. ${ }^{2}$ The influence of the spatial demand of the aryl groups at positions 2 and 4 has also been investigated. ${ }^{2,3}$ A special benefit of this versatile method is that the starting 2,4-diaryl-2,3dihydro-1,5-benzothiazepines are readily available substances by the reaction of $\alpha, \beta$-unsaturated ketones with 2-aminothiophenol. ${ }^{4}$

2-Styrylbenzothiazoles have been prepared by an acid catalyzed or by a thermally mediated ring contraction of other groups of 2,3-dihydro-1,5-benzothiazepines. ${ }^{5}$ However, these conversions cannot be utilized for the synthesis of 2,2-disubstituted benzothiazoles. It should also be mentioned that several 3-acyl-2,3-dihydrobenzothiazoles were obtained by the acylation of the corresponding 2,3-dihydrobenzothiazoles either with acyl halides or with acid anhydrides in the presence of an organic or an inorganic base. ${ }^{6}$ 
According to our knowledge, no oxidative transformation of 2,2-disubstituted 3-acyl-2,3dihydrobenzothiazoles have hitherto been published in the chemical literature. This fact prompted us to investigate the dimethyldioxirane oxidation of such benzothiazoles synthesized by us.

\section{Results and Discussion}

\section{Preparation of the 2,2-Disubstituted 3-Acetyl-2,3-dihydrobenzothiazoles}

As a continuation of our study on the ring contraction of the 2,4-diaryl-2,3-dihydro-1,5benzothiazepines to provide 2,2-disubstituted 3-acyl-2,3-dihydrobenzothiazoles under acylating conditions, we have investigated this transformation with some other substrates. Compounds 110 were allowed to react with a mixture of acetic anhydride and pyridine to afford 3-acetyl-2,3dihydro-2-phenyl-2-styrylbenzothiazoles 11-20 (Scheme 1). Structures of

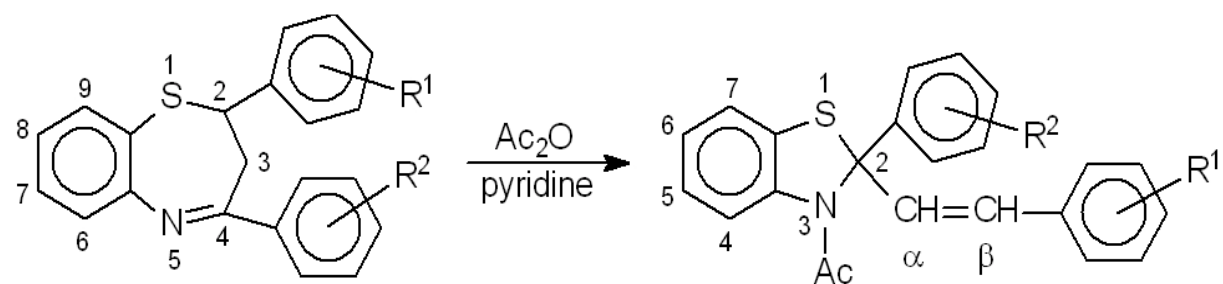

1-10

1, 11: $R^{1}=4-i P r, R^{2}=2-O A C$

2, 12: $R^{1}=2-O M e, R^{2}=2-O A C$

3, 13: $R^{1}=3,4,5-(\mathrm{OMe})_{3}, R^{2}=2-O A C$

4, 14: $R^{1}=2-C l, R^{2}=2-O A C$

5, 15: $R^{1}=3-C l, R^{2}=2-O A C$
$11-20$

6, 16: $\mathrm{R}^{1}=4-\mathrm{Cl}, \mathrm{R}^{2}=2-\mathrm{OAC}$

7, 17: $R^{1}=2,4-\mathrm{Cl}_{2}, \mathrm{R}^{2}=2-\mathrm{OAC}$

8, 18: $R^{1}=3,4-C_{2}, R^{2}=2-O A C$

9, 19: $\mathrm{R}^{1}=4-\mathrm{Cl}, \mathrm{R}^{2}=4-\mathrm{OAC}$

10, 20: $R^{1}=2,4-\mathrm{Cl}_{2}, \mathrm{R}^{2}=4-\mathrm{Me}$

\section{Scheme 1}

these new compounds were elucidated by microanalyses, IR and NMR spectroscopic measurements. In their IR spectra two $\mathrm{C}=\mathrm{O}$ bands belonging to the $\mathrm{N}$-acetyl and O-acetyl moieties were detected ( $c$. Experimental Section). In each ${ }^{1} \mathrm{H}$-NMR spectra a broad singlet signal at about $2.0 \mathrm{ppm}$ is characteristic for the $\mathrm{N}$-acetyl group. Doublet signal of the $\alpha$-proton is overlapped by the aromatic signals in all cases. However, the doublet signal of the $\beta$-proton at about $6.7 \mathrm{ppm}$ with ca. $16 \mathrm{~Hz}$ coupling constant is well separated in some instances indicating the presence of an $(E)$-disubstituted vinyl group. It is worth mentioning that the ortho-acetoxy group in the phenyl ring at position 2 of compounds $\mathbf{1 - 8}$ is without influence on the acetylation of the nitrogen atom and, therefore, on the course of the ring contraction leading to the formation of benzothiazoles 11-18. All these findings reveal that our previously introduced procedure can 
be successfully utilized for the synthesis of 2,2-disubstituted 3-acyl-2,3-dihydrobenzothiazoles substituted both at the 2-phenyl and 2-styryl groups.

\section{Oxidation Study}

Dimethyldioxirane (DMD) has been successfully utilized for the chemoselective oxidation of the sulfur atom of sulfur-containing compounds possessing carbon-carbon double bond as well. ${ }^{8}$ The above mentioned 3-acetyl-2,3-dihydrobenzothiazoles appeared to be convenient substrates to get newer insights into the scope and limitation of the utility of the dimethyldioxirane for a chemoselective oxidation of the sulfur atom of compounds with various sites of oxidation. For

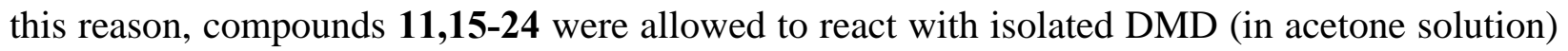
and their 1,1-dioxides $\mathbf{2 5 - 3 5}$ were obtained as sole isolable product in each case (Scheme 2).

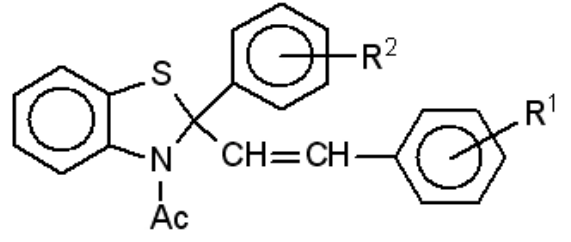

$11,15-24$

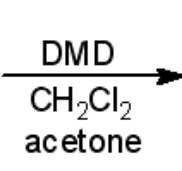

21, 25: $\mathrm{R}^{1}=\mathrm{R}^{2}=\mathrm{H}$

22, 26: $R^{1}=H, R^{2}=2-O A c$

23, 27: $R^{1}=H, R^{2}=4-O A c$

24, 28: $R^{1}=H, R^{2}=4-O M e$

11, 29: $R^{1}=4-i P r, R^{2}=2-O A c$

15, 30: $\mathrm{R}^{1}=3-\mathrm{Cl}, \mathrm{R}^{2}=2-\mathrm{OAC}$

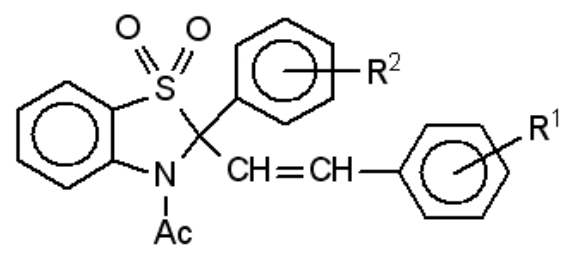

25-35

16, 31: $\mathrm{R}^{1}=4-\mathrm{Cl}, \mathrm{R}^{2}=2-\mathrm{OAC}$

17, 32: $R^{1}=2,4-\mathrm{Cl}_{2}, \mathrm{R}^{2}=2-\mathrm{OAC}$

18, 33: $R^{1}=3,4-\mathrm{Cl}_{2}, \mathrm{R}^{2}=2-\mathrm{OAc}$

19, 34: $\mathrm{R}^{1}=4-\mathrm{Cl}, \mathrm{R}^{2}=4-\mathrm{OAC}$

20, 35: $R^{1}=2,4-\mathrm{Cl}_{2}, \mathrm{R}^{2}=4-\mathrm{Me}$

\section{Scheme 2}

Structure elucidation of sulfones $\mathbf{2 5 - 3 5}$ has been performed by elemental analyses and spectroscopic measurements. In their ${ }^{1} \mathrm{H}-\mathrm{NMR}$ spectra the presence of the doublets with $\mathrm{ca} .16$ $\mathrm{Hz}$ coupling constant value assigned to the $\alpha-\mathrm{H}$ and $\beta-\mathrm{H}$ atoms unequivocally prove that the carbon-carbon double bond was not oxidized even by a large excess (4 equivalents) of DMD.

Electron impact $(70 \mathrm{eV}$ ) mass spectra of compounds 25-35 are very similar. A very weak molecular ion (approx. 0.1\%) could be detected only in some cases. However, an $\mathrm{m} / \mathrm{z} 43$ $\left(\mathrm{C}_{2} \mathrm{H}_{3} \mathrm{O}\right)$ is the base peak in all cases. The major fragmentation involves the loss of two oxygen atoms and $\mathrm{CH}_{2} \mathrm{CO}$ unit resulting in the formation of an M-74 ion.

If dimethyldioxirane is used as an oxidant, the nitrogen atom can also be a site of oxidation as observed for various nitrogen-containing heterocyclic compounds. ${ }^{9}$ Here, N-oxidation did not take place and finally only the appropriate sulfone could be detected which is in agreement with the previous results indicating that the amide nitrogen is not oxidized by DMD. ${ }^{9 a, g, 10}$ Dimethyldioxirane oxidation of the sulfur atom usually results in the formation of sulfoxide or sulfone depending on the amount of the DMD. We have detected a new product in the reaction 
mixture by thin layer chromatography (TLC) which disappeared on the progress of the oxidation and finally the appropriate sulfone could only be detected and isolated. We were unable to isolate sulfoxide even by using less than one equivalent of DMD. In such a case the starting material and the sulfone were the major components in the reaction mixture. Formerly we have observed that if 1-thiochromones were oxidized with $\mathrm{DMD}$, the sulfoxides were more reactive than the parent sulfides toward dimethyldioxirane. ${ }^{8 \mathrm{~d}}$ This reactivity pattern is quite unique since sulfides are generally much more reactive that the sulfoxides on a DMD oxidation. ${ }^{11}$ Now, we observed the same phenomenon on the DMD oxidaton of 3-acetyl-2,3-dihydro-2-phenyl-2styrylbenzothiazoles.

In conclusion, we have succeeded in a completely chemoselective oxidation of the sulfur atom of the above mentioned benzothiazoles without the oxidation of the carbon-carbon double bond and the nitrogen atom. It means that the dimethyldioxirane is the oxidant of choice if the aim is to prepare the sulfones of such benzothiazoles. Our present study provides additional examples for the unexpected reaction pattern of the DMD oxidation of sulfoxides and the parent sulfides by using hitherto non-investigated substrates.

\section{Experimental Section}

General Procedures. Melting points were determined with a Koffler hot-stage apparatus and are uncorrected. NMR spectra were recorded on a Varian Gemini 200 spectrometer at 200/50 MHz in $\mathrm{CDCl}_{3}$ (internal standard TMS, $\delta=0.0 \mathrm{ppm}$ ) at room temperature. Mass spectra were recorded on a VG TRIO-2 instrument in the EI mode at $70 \mathrm{eV}$. The IR spectra (KBr discs) were obtained with a Perkin-Elmer 16 PC instrument. Elemental analyses were measured in-house with a Carlo Erba 1106 EA apparatus. TLC was performed on Kieselgel $60 \mathrm{~F}_{254}$ (Merck) layer using 1,2dichloroethane or hexane:acetone $(7: 3 \mathrm{v} / \mathrm{v})$ as eluents. Starting materials 1-10 and 21-24 were synthesized as described by us. ${ }^{1,2,4 c, d, i}$ Dimethyldioxirane (as an acetone solution) was prepared as described $^{7}$ and its peroxide content was determined iodometrically.

General procedure for the preparation of 3-acetyl-2,3-dihydro-2-aryl-2styrylbenzothiazoles (11-20). A mixture of 2,4-diaryl-2,3-dihydro-1,5-benzothiazepines 1-10 (10.0 mmoles), acetic anhydride $(25 \mathrm{~mL})$ and anhydrous pyridine $(10.0 \mathrm{~mL})$ was refluxed for 4 $\mathrm{h}$ and then poured onto crushed ice. The crude oily product was extracted with methylene chloride ( 4 x $50 \mathrm{~mL}$ ). The separated organic phase was washed with brine and dried over $\mathrm{CaCl}_{2}$. The solvent was evaporated under reduced pressure and the residue was purified by column chromatography using silica gel (Merck) and 1,2-dichloroethane as eluent to obtain substances 11-20.

3-Acetyl-2,3-dihydro-2-(2-acetoxyphenyl)-2-(4-isopropylstyryl)benzothiazole (11). This compound was obtained as white plates in 69\% yield, mp 95-96 ${ }^{\circ} \mathrm{C}$; IR: vC=O 1767 and 1671 
$\mathrm{cm}^{-1}$; ${ }^{1} \mathrm{H}-\mathrm{NMR}(\delta): 1.22$ (6H, d, $\left.J=6.9 \mathrm{~Hz}, \mathrm{CH}\left(\mathrm{CH}_{3}\right)_{2}\right), 1.88$ (3H, s, OAc), 2.08 (3H, s, NAc), $2.89\left(1 \mathrm{H}, \mathrm{m}, \mathrm{C} \underline{\mathrm{H}}\left(\mathrm{CH}_{3}\right)_{2}\right)$, 6.75-7.50 (m, $\alpha-\mathrm{H}, \beta-\mathrm{H}+12$ arom. $\left.\mathrm{H}\right) ;{ }^{13} \mathrm{C}-\mathrm{NMR}(\delta): 20.5$ (OAc), 23.6 $\left(\mathrm{CH}\left(\mathrm{CH}_{3}\right)_{2}\right), 25.7$ (NAc), $33.7\left(\underline{\mathrm{CH}}\left(\mathrm{CH}_{3}\right)_{2}\right), 168.9$ (OAc), 170.2 (NAc); Anal. Calcd. for $\mathrm{C}_{28} \mathrm{H}_{27} \mathrm{NO}_{3} \mathrm{~S}$ : C, 73.50; H, 5.95; N, 3.06. Found: C, 73.53; H, 5.93; N, 3.08.

3-Acetyl-2,3-dihydro-2-(2-acetoxyphenyl)-2-(2-methoxystyryl)benzothiazole (12). This compounds was isolated as white needles in 65\% yield, mp $125-126{ }^{\circ} \mathrm{C}$; IR: vC=O 1767 and $1672 \mathrm{~cm}^{-1}$; ${ }^{1} \mathrm{H}-\mathrm{NMR}(\delta): 1.94$ (3H, s, OAc), 2.08 (3H, s, NAc), 3.87 (3H, s, OMe), 6.72-8.01 (m, $\alpha-\mathrm{H}, \beta-\mathrm{H}+12$ arom. H); ${ }^{13} \mathrm{C}-\mathrm{NMR}(\delta)$ : 20.6 (OAc), 25.7 (NAc), 55.2 (OMe), 168.9 (OAc), 170.2 (NAc); Anal. Calcd. for $\mathrm{C}_{26} \mathrm{H}_{23} \mathrm{NO}_{4} \mathrm{~S}$ : C, 70.10; H, 5.20; N, 3.14. Found: C, 70.14; H, 5.23; N, 3.12.

3-Acetyl-2,3-dihydro-2-(2-acetoxyphenyl)-2-(3,4,5-trimethoxystyryl)benzothiazole (13). This substance was prepared as white plates in 61\% yield, mp 182-183 ${ }^{\circ} \mathrm{C}$; IR: vC=O 1762 and 1673 $\mathrm{cm}^{-1}$; ${ }^{1} \mathrm{H}-\mathrm{NMR}(\delta): 1.92$ (3H, s, OAc), 2.07 (3H, s, NAc), 3.83 (3H, s, OMe), 3.90 (6H, s, 2OMe), 6.65-7.89 (m, $\alpha-\mathrm{H}, \beta-\mathrm{H}+10$ arom. $\mathrm{H}$ ); ${ }^{13} \mathrm{C}-\mathrm{NMR}(\delta)$ : 20.6 (OAc), 25.6 (NAc), 56.0 (2OMe), 60.7 (OMe), 168.9 (OAc), 170.0 (NAc); Anal. Calcd. for $\mathrm{C}_{28} \mathrm{H}_{27} \mathrm{NO}_{6} \mathrm{~S}$ : C, 66.52; $\mathrm{H}$, 5.38; N, 2.77. Found: C, 66.56; H, 5.36; N, 2.75.

3-Acetyl-2,3-dihydro-2-(2-acetoxyphenyl)-2-(2-chlorostyryl)benzothiazole (14). This compound was isolated as white plates in 76\% yield, mp 162-163 ${ }^{\circ} \mathrm{C}$; IR: vC=O 1765 and 1676 $\mathrm{cm}^{-1}$; ${ }^{1} \mathrm{H}-\mathrm{NMR}(\delta): 1.92$ (3H, s, OAc), 2.09 (3H, s, NAc), $6.70(1 \mathrm{H}, \mathrm{d}, J=15.7 \mathrm{~Hz}, \quad \beta-\mathrm{H}), 7.00-$ 7.89 (m, $\alpha-\mathrm{H}+12$ arom. H); ${ }^{13} \mathrm{C}-\mathrm{NMR}(\delta)$ : 20.5 (OAc), 25.3 (NAc), 168.7 (OAc), 169.8 (NAc); Anal. Calcd. for $\mathrm{C}_{25} \mathrm{H}_{20} \mathrm{ClNO}_{3} \mathrm{~S}$ : C, 66.74; H, 4.48; N, 3.11. Found C, 66.78; H, 4.46; N, 3.12 .

3-Acetyl-2,3-dihydro-2-(2-acetoxyphenyl)-2-(3-chlorostyryl)benzothiazole (15). This substance was obtained as pale yellow plates in 63\% yield, mp $145-146{ }^{\circ} \mathrm{C}$; IR: vC=O 1766 and $1666 \mathrm{~cm}^{-1}$; ${ }^{1} \mathrm{H}-\mathrm{NMR}(\delta): 1.90$ (3H, s, OAc), 2.06 (3H, s, NAc), 6.71 (1H, d, $\left.J=15.8 \mathrm{~Hz}, \beta-\mathrm{H}\right)$, 6.91-7.87 (m, $\alpha-\mathrm{H}+12$ arom. H); ${ }^{13} \mathrm{C}-\mathrm{NMR}(\delta): 20.5$ (OAc), 25.5 (NAc), 168.8 (OAc), 169.9 (NAc); Anal. Calcd. for $\mathrm{C}_{25} \mathrm{H}_{20} \mathrm{ClNO}_{3} \mathrm{~S}$ : C, 66.74; H, 4.48; N, 3.11. Found: C, 66.79; H, 4.50; N, 3.14 .

3-Acetyl-2,3-dihydro-2-(2-acetoxyphenyl)-2-(4-chlorostyryl)benzothiazole (16). This compound was obtained as white plates in 71\% yield, mp 153-154 ${ }^{\circ} \mathrm{C}$; IR: vC=O 1772 and 1679 $\mathrm{cm}^{-1}$; ${ }^{1} \mathrm{H}-\mathrm{NMR}(\delta): 1.89$ (3H, s, OAc), 2.06 (3H, s, NAc), 6.73(1H, d, $\left.J=15.7 \mathrm{~Hz}, \beta-\mathrm{H}\right), 6.85-$ 7.86 (m, $\alpha-\mathrm{H}+12$ arom. $\mathrm{H}$ ); ${ }^{13} \mathrm{C}-\mathrm{NMR}(\delta): 20.5$ (OAc), 25.5 (NAc), 168.8 (OAc), 169.8 (NAc); Anal. Calcd. for $\mathrm{C}_{25} \mathrm{H}_{20} \mathrm{ClNO}_{3} \mathrm{~S}$ : C, 66.74; H, 4.48; N, 3.11. Found, C, 66.71; H, 4.49; N, 3.09.

3-Acetyl-2,3-dihydro-2-(2-acetoxyphenyl)-2-(2,4-dichlorostyryl)benzothiazole (17). This compound was prepared as pale yellow needles in 74\% yield, mp 109-110 ${ }^{\circ} \mathrm{C}$; IR: vC=O 1765 and $1668 \mathrm{~cm}^{-1}$; ${ }^{1} \mathrm{H}-\mathrm{NMR}(\delta): 1.92$ (3H, s, OAc), 2.12 (3H, s, NAc), 6.70 (1H, d, $J=15.7 \mathrm{~Hz}, \beta-$ $\mathrm{H})$, 6.98-7.80 (m, $\alpha-\mathrm{H}+11$ arom. $\mathrm{H}$ ); ${ }^{13} \mathrm{C}-\mathrm{NMR}(\delta)$ : 20.5 (OAc), 25.2 (NAc), 168.7 (OAc), 169.6 (NAc); Anal. Calcd. for $\mathrm{C}_{25} \mathrm{H}_{19} \mathrm{Cl}_{2} \mathrm{NO}_{3} \mathrm{~S}$ : C, 61.99; H, 3.95; N, 2.89. Found: C, 61.95; H, 3.93; $\mathrm{N}, 2.91$.

3-Acetyl-2,3-dihydro-2-(2-acetoxyphenyl)-2-(3,4-dichlorostyryl)benzothiazole (18). This substance was isolated as pale yellow plates in 83\% yield, mp 170-171 ${ }^{\circ} \mathrm{C}$; IR: vC=O 1765 and 
$1664 \mathrm{~cm}^{-1}$; ${ }^{1} \mathrm{H}-\mathrm{NMR}(\delta): 1.95$ (3H, s, OAc), 2.04 (3H, s, NAc), 6.70 (1H, d, $\left.15.7 \mathrm{~Hz}, \beta-\mathrm{H}\right), 6.92-$ 7.90 (m, $\alpha-\mathrm{H}+11$ arom. H); ${ }^{13} \mathrm{C}-\mathrm{NMR}(\delta): 20.5$ (OAc), 25.3 (NAc), 168.7 (OAc), 169.7 (NAc); Anal. Calcd. for $\mathrm{C}_{25} \mathrm{H}_{19} \mathrm{Cl}_{2} \mathrm{NO}_{3} \mathrm{~S}$ : C, 61.99; H, 3.95; N, 2.89. Found: C, 62.02; H, 3.96; N, 2.86.

3-Acetyl-2,3-dihydro-2-(4-acetoxyphenyl)-2-(4-chlorostyryl)benzothiazole $\quad$ (19). This compound was prepared as white needles in 72\% yield, mp 89-90 ${ }^{\circ} \mathrm{C}$; IR: vC=O 1760 and 1671 $\mathrm{cm}^{-1}$; ${ }^{1} \mathrm{H}-\mathrm{NMR}(\delta): 2.04$ (3H, s, NAc), 2.31 (3H, s, OAc), 6.50 (1H, d, $\left.J=15.8 \mathrm{~Hz}, \beta-\mathrm{H}\right), 6.90-$ 7.71 (m, $\alpha-\mathrm{H}+12$ arom. H); ${ }^{13} \mathrm{C}-\mathrm{NMR}(\delta): 20.9$ (OAc), 25.7 (NAc), 169.3 (OAc), 170.0 (NAc); Anal. Calcd. for $\mathrm{C}_{25} \mathrm{H}_{20} \mathrm{ClNO}_{3} \mathrm{~S}$ : C, 66.74; H, 4.48; N, 3.11. Found: C, 66.70; H, 4.50; N, 3.14.

3-Acetyl-2,3-dihydro-2-(2,4-dichlorostyryl)-2-(4-methylphenyl)benzothiazole (20). This substance was obtained as white plates in 67\% yield, mp 192-193 ${ }^{\circ} \mathrm{C}$; IR: vC=O $1667 \mathrm{~cm}^{-1} ;{ }^{1} \mathrm{H}-$

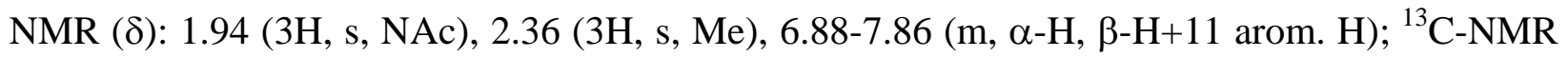

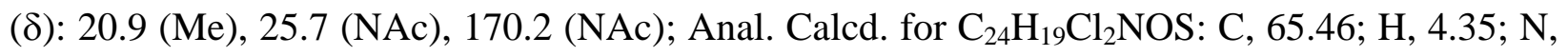
3.18. Found: C, 65.42; H, 4.33; N, 3.16.

General Procedure for the Dimethyldioxirane Oxidation of 3-Acetyl-2,3-dihydrobenzothiazoles (11, 15-24). Compounds 11,15-24 (2.5 mmoles) were dissolved in anhydrous methylene chloride (10.0 mL), dimethyldioxirane (10.0 mmoles as $0.05 \mathrm{M}$ acetone solution) was added and the mixture was allowed to stand at room temperature for $16 \mathrm{~h}$. The solvent was evaporated under reduced pressure and the residue was crystallized from methanol to afford sulfones 25-35.

3-Acetyl-2,3-dihydro-2-phenyl-2-styrylbenzothiazole 1,1-dioxide (25). This compound was isolated as white needles in 84\% yield, mp 133-134 ${ }^{\circ} \mathrm{C}$; IR: vC=O $1692 \mathrm{~cm}^{-1} ;{ }^{1} \mathrm{H}-\mathrm{NMR}(\delta): 1.80$ (3H, s, NAc), 6.63 (1H, d, J = 16 Hz, $\beta-H), 7.12$ (1H, d, $J=16$ Hz, $\alpha-H)$, 7.27-8.67 (m, 14 arom. $\mathrm{H}) ;{ }^{13} \mathrm{C}-\mathrm{NMR}(\delta)$ : 26.8 (NAc), 171.5 (NAc); Anal. Calcd. for $\mathrm{C}_{23} \mathrm{H}_{19} \mathrm{NO}_{3} \mathrm{~S}$ : C, 70.94; H, 4.92; N, 3.59. Found: C, 70.90; H, 4.94; N, 3.61.

3-Acetyl-2,3-dihydro-2-(2-acetoxyphenyl)-2-styrylbenzothiazole 1,1-dioxide (26). This substance was obtained as white needles in 81\% yield, mp 157-158 ${ }^{\circ} \mathrm{C}$; IR: $v \mathrm{C}=\mathrm{O} 1768$ and 1690 $\mathrm{cm}^{-1}$; ${ }^{1} \mathrm{H}-\mathrm{NMR}(\delta): 1.80$ (3H, s, NAc), 1.92 (3H, s, OAc), 6.68 (1H, d, $\left.J=16 \mathrm{~Hz}, \beta-\mathrm{H}\right), 7.04-$ 8.78 (m, $\alpha-\mathrm{H}+13$ arom. H); ${ }^{13} \mathrm{C}-\mathrm{NMR}(\delta): 20.7$ (OAc), 26.3 (NAc), 168.1 (OAc), 171. (NAc); Anal. Calcd. for $\mathrm{C}_{25} \mathrm{H}_{21} \mathrm{NO}_{5} \mathrm{~S}$ : C 67.11; H, 4.73; N, 3.13. Found: C, 67.08; H, 4.75; N, 3.15 .

3-Acetyl-2,3-dihydro-2-(4-acetoxyphenyl)-2-styrylbenzothiazole 1,1-dioxide (27). This compound was prepared as white needles in 88\% yield, mp 134-135 ${ }^{\circ} \mathrm{C}$; IR: vC=O 1754 and $1690 \mathrm{~cm}^{-1} ; \quad{ }^{1} \mathrm{H}-\mathrm{NMR}(\delta): 1.88$ (3H, s, NAc), 2.30 (3H, s, OAc), 6.67 (1H, d, J = $\left.16 \mathrm{~Hz}, \beta-\mathrm{H}\right)$, 7.08 (1H, d, $J=16 \mathrm{~Hz}, \alpha-\mathrm{H}), 7.22-8.61$ (m, 13 arom. H); ${ }^{13} \mathrm{C}-\mathrm{NMR}(\delta): 20.9$ (OAc), 26.8 (NAc), 168.9 (OAc), 171.3 (NAc); Anal. Calcd. for $\mathrm{C}_{25} \mathrm{H}_{21} \mathrm{NO}_{5} \mathrm{~S}: \mathrm{C}, 67.11 ; \mathrm{H}, 4.73$; N, 3.13. Found: C, 67.15; H, 4.71; N, 3.15.

3-Acetyl-2,3-dihydro-2-(4-methoxyphenyl)-2-styrylbenzothiazole 1,1-dioxide (28). This substance was obtained as white plates in 83\% yield, mp $168-169{ }^{\circ} \mathrm{C}$; IR: vC=O $1686 \mathrm{~cm}^{-1} ;{ }^{1} \mathrm{H}-$

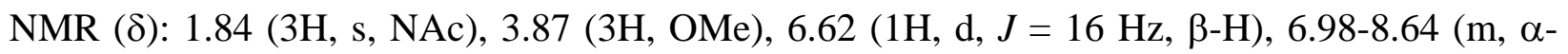
$\mathrm{H}+13$ arom. $\mathrm{H}$ ); ${ }^{13} \mathrm{C}-\mathrm{NMR}(\delta)$ : 26.8 (NAc), 55.3 (OMe), 171.6 (NAc); Anal. Calcd. for $\mathrm{C}_{24} \mathrm{H}_{21} \mathrm{NO}_{4} \mathrm{~S}$ : C, 68.72; H, 5.05; N, 3.34. Found: C, 68.76; H, 5.07; N, 3.31. 
3-Acetyl-2,3-dihydro-2-(2-acetoxyphenyl)-2-(4-isopropylstyryl)benzothiazole 1,1-dioxide (29). This compound was prepared as white needles in $94 \%$ yield, mp $157-158{ }^{\circ} \mathrm{C}$; IR: vC=O 1671 and $1689 \mathrm{~cm}^{-1}$; ${ }^{1} \mathrm{H}-\mathrm{NMR}(\delta): 1.24$ (6H, d, $\left.J=6.8 \mathrm{~Hz}, \mathrm{CH}\left(\mathrm{CH}_{3}\right)_{2}\right), 1.80$ (3H, s, NAc), 1.90 (3H, s, OAc), $2.91\left(1 \mathrm{H}, \mathrm{m}, \mathrm{C} \underline{\mathrm{H}}\left(\mathrm{CH}_{3}\right)_{2}\right), 6.68(1 \mathrm{H}, \mathrm{d}, J=16 \mathrm{~Hz}, \beta-\mathrm{H}), 7.04-8.77(\mathrm{~m}, \quad \alpha-\mathrm{H}+12$ arom. H); ${ }^{13} \mathrm{C}-\mathrm{NMR}(\delta): 20.6$ (OAc), $23.6\left(\mathrm{CH}\left(\mathrm{CH}_{3}\right)_{2}\right), 26.2(\mathrm{NAc}), 33.7\left(\underline{\mathrm{CH}}\left(\mathrm{CH}_{3}\right)_{2}\right), 168.1$ (OAc), 171.1 (NAc); Anal. Calcd. for $\mathrm{C}_{28} \mathrm{H}_{27} \mathrm{NO}_{5} \mathrm{~S}$ : C, 68.69; H, 5.56; N, 2.86. Found: C, 68.65; H, 5.54; N, 2.84.

3-Acetyl-2,3-dihydro-2-(2-acetoxyphenyl)-2-(3-chlorostyryl)benzothiazole 1,1-dioxide (30). This compound was obtained as pale yellow needles in 91\% yield, mp 156-157 ${ }^{\circ} \mathrm{C}$; IR: vC=O 1761 and $1685 \mathrm{~cm}^{-1}$; ${ }^{1} \mathrm{H}-\mathrm{NMR}(\delta): 1.82$ (NAc), 1.91 (OAc), 6.63 (1H, d, J = $15.8 \mathrm{~Hz}, \beta-\mathrm{H}$ ), 7.108.73 (m, $\alpha-\mathrm{H}+12$ arom. $\mathrm{H}$ ); ${ }^{13} \mathrm{C}-\mathrm{NMR}(\delta): 20.8$ (OAc), 26.3 (NAc), 168.1 (OAc), 170.9 (NAc); Anal. Calcd. for $\mathrm{C}_{25} \mathrm{H}_{20} \mathrm{ClNO}_{5} \mathrm{~S}$ : C, 62.31; H, 4.18; N, 2.90. Found: C, 62.35; H, 4.20; N, 2.92.

3-Acetyl-2,3-dihydro-2-(2-acetoxyphenyl)-2-(4-chlorostyryl)benzothiazole 1,1-dioxide (31). This substance was prepared as pale yellow plates in 94\% yield, mp 114-115 ${ }^{\circ} \mathrm{C}$; IR: vC=O 1764 and $1688 \mathrm{~cm}^{-1}$; ${ }^{1} \mathrm{H}-\mathrm{NMR}(\delta): 1.80$ (3H, s, NAc), 1.91 (3H, s, OAc), 6.64 (1H, d, $J=15.8 \mathrm{~Hz}, \beta-$ $\mathrm{H}$ ), 7.05-8.75 (m, $\alpha-\mathrm{H}+12$ arom. $\mathrm{H}$ ); ${ }^{13} \mathrm{C}-\mathrm{NMR}(\delta)$ : 20.6 (OAc), 26.2 (NAc), 168.1 (OAc), 170.9 (NAc); Anal. Calcd. for $\mathrm{C}_{25} \mathrm{H}_{20} \mathrm{ClNO}_{5} \mathrm{~S}$ : C, 62.31; H, 4.18; N, 2.90. Found: C, 62.27; H, 4.16; N, 2.89 .

3-Acetyl-2,3-dihydro-2-(2-acetoxyphenyl)-2-(2,4-dichlorostyryl)benzothiazole 1,1-dioxide (32). This compound was obtained as pale yellow plates in $78 \%$ yield, mp $161-162{ }^{\circ} \mathrm{C}$; IR: $v \mathrm{C}=\mathrm{O}$ 1763 and $1693 \mathrm{~cm}^{-1} ;{ }^{1} \mathrm{H}-\mathrm{NMR}(\delta): 1.78$ (3H, s, NAc), 1.93 (3H, s, OAc), $6.60(1 \mathrm{H}, \mathrm{d}, J=15.8$ $\mathrm{Hz}, \beta-\mathrm{H}), 7.10$ (1H, d, $J=15.8 \mathrm{~Hz}, \alpha-\mathrm{H}), 7.28-8.78$ (m, 11 arom. H); ${ }^{13} \mathrm{C}-\mathrm{NMR}(\delta): 20.7$ (OAc), 26.3 (NAc), 168.1 (OAc), 170.8 (NAc); Anal. Calcd. for $\mathrm{C}_{25} \mathrm{H}_{19} \mathrm{Cl}_{2} \mathrm{NO}_{5} \mathrm{~S}: \mathrm{C}, 58.15 ; \mathrm{H}, 3.71 ; \mathrm{N}$, 2.71. Found: C, 58.17; H, 3.69; N, 2.73.

3-Acetyl-2,3-dihydro-2-(2-acetoxyphenyl)-2-(3,4-dichlorostyryl)benzothiazole 1,1-dioxide (33). This substance was isolated as pale yellow needles in $81 \%$ yield, mp $146-147^{\circ} \mathrm{C}$; IR: vC=O 1758 and $1672 \mathrm{~cm}^{-1}$; ${ }^{1} \mathrm{H}-\mathrm{NMR}(\delta): 1.81$ (3H, s, NAc), 1.92 (3H, s, OAc), 6.59 (1H, d, $J=16 \mathrm{~Hz}$, $\beta-\mathrm{H}), 7.11$ (1H, d, $J=16 \mathrm{~Hz}, \alpha-\mathrm{H}), 7.23-8.73$ (M, 11 arom. H); ${ }^{13} \mathrm{C}-\mathrm{NMR}(\delta): 20.7$ (OAc), 26.3 (NAc), 168.0 (OAc), 170.8 (NAc); Anal. Calcd. for $\mathrm{C}_{25} \mathrm{H}_{19} \mathrm{Cl}_{2} \mathrm{NO}_{5} \mathrm{~S}$ : C, 58.15; H, 3.71; N, 2.71. Found: C, 58.11; H, 3.74; N, 2.68.

3-Acetyl-2,3-dihydro-2-(4-acetoxyphenyl)-2-(4-chlorostyryl)benzothiazole 1,1-dioxide (34). This compound was isolated as white plates in 84\% yield, mp 120-121 ${ }^{\circ} \mathrm{C}$; IR: vC=O 1762 and $1690 \mathrm{~cm}^{-1}$; ${ }^{1} \mathrm{H}-\mathrm{NMR}(\delta): 1.79$ (3H, s, NAc), 1.92 (3H, s, OAc), 6.59 (1H, d, $\left.J=15.7 \mathrm{~Hz}, \beta-\mathrm{H}\right)$, 7.10-8.18 (m, $\alpha-\mathrm{H}+12$ arom. H); ${ }^{13} \mathrm{C}-\mathrm{NMR}(\delta)$ : 20,7 (OAc), 26.0 (NAc), 168.2 (OAc), 171.0 (NAc); Anal. Calcd. for $\mathrm{C}_{25} \mathrm{H}_{20} \mathrm{ClNO}_{5} \mathrm{~S}$ : C, 62.31; H, 4.18; N, 2.90. Found: C, 62.29; H, 4.20; N, 2.87.

3-Acetyl-2,3-dihydro-2-(2,4-dichlorostyryl)-2-(4-methylphenyl)benzothiazole 1,1-dioxide (35). This substance was obtained as pale yellow plates in $78 \%$ yield, mp 174-175 ${ }^{\circ} \mathrm{C}$; IR: $v \mathrm{C}=\mathrm{O}$ $1693 \mathrm{~cm}^{-1}$; ${ }^{1} \mathrm{H}-\mathrm{NMR}(\delta): 1.85$ (3H, s, NAc), 2.40 (3H, s, Me), 6.96 (1H, d, $\left.J=15.9 \mathrm{~Hz}, \beta-\mathrm{H}\right)$, $7.10(1 \mathrm{H}, \mathrm{d}, J=15.9 \mathrm{~Hz}, \alpha-\mathrm{H}), 7.23-8.63$ (m, 11 arom. H); ${ }^{13} \mathrm{C}-\mathrm{NMR}(\delta): 21.1(\mathrm{Me}), 26.7$ 
(NAc), 171.4 (NAc); Anal. Calcd. for $\mathrm{C}_{25} \mathrm{H}_{19} \mathrm{Cl}_{2} \mathrm{NO}_{3} \mathrm{~S}$ : C, 61.03; H, 4.05; N, 2.96. Found: C, 61.06; H, 4.07; N, 2.94.

\section{Acknowledgements}

The present study was sponsored by the Hungarian National Research Foundation (Grant Nos. OTKA T029171 and T034123) for which our gratitude is expressed. Technical assistance of Mrs. M. Nagy is highly appreciated.

\section{References}

1. Tóth, G.; Lévai, A.; Balázs, B.; Simon, A. Liebigs Ann./Recueil 1997, 995.

2. Lévai, A. Monatsh. Chem. 1998, 129, 909.

3. Lévai, A. Heterocycl. Commun. 2002, 8, 375.

4. (a) Stephens, W. D.; Field, L. J. Org. Chem. 1959, 24, 1576. (b) Hideg, K.; Hankovszky, O. Acta Chim. Acad. Sci. Hung. 1968, 56, 405. (c) Lévai, A.; Bognár R. Acta Chim. Acad. Sci. Hung. 1976, 88, 293. (d) Lévai, A.; Bognár, R. Acta Chim.. Acad. Sci. Hung. 1977, 92, 415. (e) Lévai, A. Pharmazie 1979, 34, 439. (f) Lévai, A.; Bognár, R.; Kajtár, J. Acta Chim. Acad. Sci. Hung. 1980, 103, 27. (g) Lévai, A. Pharmazie 1981, 36, 449. (h) Lévai, A. Trends Heterocycl. Chem. 1985, 4, 51. (i) Lévai, A. Heterocycl. Commun. 1999, 5, 359. (j) Lévai, A. J. Heterocycl. Chem. 2000, 37, 199.

5. (a) Mills, W. H.; Whitworth, J. B. J. Chem. Soc. 1927, 2738. (b) Wilhelm, M.; Schmidt, P. Helv. Chim. Acta 1970, 53, 1697. (c) Kaupp, G.; Gründken, E.; Matthies, D. Chem. Ber. 1986, 119, 3109. (d) Kaupp, G.; Matthies, D. Chem. Ber. 1987, 120, 1741.

6. (a) Bognár, R.; Kolodynska, Z.; Somogyi, L.; Györgydeák, Z.; Szilágyi, L.; Nemes, É. N. Acta Chim. Acad. Sci. Hung. 1969, 62, 65. (b) Hori, M.; Kataoka, T.; Shimizu, H.; Imari, Y. Heterocycles 1978, 10, 17. (c) Hori, M.; Kataoka, T.; Shimizu, H.; Imai, Y. Chem. Pharm. Bull. 1979, 27, 1973. (d) Hori, M.; Kataoka, T.; Shimizu, H.; Imai, Y. Chem. Pharm. Bull. 1979, 27, 1982. (e) Trapani, G.; Reho, A.; Latrofa, A.; Liso, G. Synthesis 1988, 84.

7. Adam, W.; Bialas, L.; Hadjiarapoglou, L. Chem. Ber. 1991, 124, 2377.

8. (a) Adam, W.; Golsch, D.; Hadjiarapoglou, L.; Lévai, A.; Nemes, C.; Patonay, T. Tetrahedron 1994, 50, 13113. (b) Gunda, E. T.; Tamás, L.; Sályi, S.; Nemes, C.; Sztaricskai, F. Tetrahedron Lett. 1995, 36, 7111. (c) Lautens, M.; Fillion, E.; Sampat, M. Tetrahedron Lett. 1998, 39, 1501. (d) Patonay, T.; Adam, W.; Lévai, A.; Kövér P.; Németh, M.; Peters, E. M.; Peters, K. J. Org. Chem. 2001, 66, 2275.

9. (a) Murray, R. W.; Singh, M. Tetrahedron Lett. 1988, 29, 4677. (b) Adam, W.; Golsch, D. Angew. Chem. Int. Ed. Engl. 1993, 32, 737. (c) Brik, M. E. Tetrahedron Lett. 1995, 36, 5519. (d) Adam, W.; Briviba, K.; Duschek, F.; Golsch, D.; Kiefer, W.; Sies, H. J. Chem. 
Soc., Chem. Commun. 1995, 1831. (e) Ferrer, M.; Sánchez-Baeza, F.; Messeguer, A.; Diez, A.; Rubiralta, M. J. Chem. Soc., Chem. Commun. 1995, 293. (f) Ferrer, M.; Sánchez-Baeza, F.; Messeguer, A. Tetrahedron 1997, 53, 15877. (g) Éles, J.; Kalaus, G.; Lévai, A.; Greiner, I.; Kajtár-Peredy, M.; Szabó, P.; Szabó, L.; Szántay, C. J. Heterocycl. Chem. 2002, 39, 767.

10. Adam, W.; Lévai, A.; Mérour, J. Y.; Nemes, C.; Patonay, T. Synthesis 1997, 268.

11. (a) Murray, R. W.; Jeyaraman, R. J. Org. Chem. 1985, 50, 2847. (b) Murray, R. W.; Jeyaraman, R.; Krisna Pillay, M. J. Org. Chem. 1987, 52, 740. 